\title{
La lutte entre image et imagination : Dario Fo joue (et dessine) Johan Padan
}

La lotta fra immagine e immaginazione. Dario Fo recita (e disegna) Johan Padan The struggle between image and imagination. Dario Fo plays (and draws) Johan Padan

\section{Federica Tummillo}

\section{OpenEdition}

\section{Journals}

Édition électronique

URL : http://journals.openedition.org/cei/563

DOI : 10.4000/cei.563

ISSN : 2260-779X

\section{Éditeur}

UGA Éditions/Université Grenoble Alpes

\section{Édition imprimée}

Date de publication : 15 mars 2012

Pagination : 245-254

ISBN : 978-2-84310-222-6

ISSN : $1770-9571$

Référence électronique

Federica Tummillo, "La lutte entre image et imagination : Dario Fo joue (et dessine) Johan Padan », Cahiers d'études italiennes [En ligne], 14 | 2012, mis en ligne le 15 septembre 2013, consulté le 28 mars 2021. URL : http://journals.openedition.org/cei/563 ; DOI : https://doi.org/10.4000/cei.563 


\title{
LA LUTTE ENTRE IMAGE ET IMAGINATION : DARIO FO JOUE (ET DESSINE) JOHAN PADAN
}

\author{
Federica Tummillo \\ Université Stendhal - Grenoble 3
}

En 1991 Dario Fo découvre finalement l'Amérique sur scène. Si en I963 le rôle de Christophe Colomb dans Isabelle, trois caravelles et un charlatan, l'avait obligé de rester à la cour d'Isabelle la Catholique pour mettre à nu l'obscénité du pouvoir politique, trente ans plus tard le personnage de Johan Padan lui permet de tenter sa fortune à bord d'une caravelle pour échapper au Tribunal de l'Inquisition.

Pendant un voyage merveilleux et terrible, Johan, un homme quelconque, survivra au naufrage sur le dos d'un porc, fera la rencontre des tribus indigènes de l'Amérique centrale, il sera à la fois marin, prophète, chaman, bouffon, guide révolutionnaire, homme qui vieillit en paix.

Le monologue Johan Padan a la descoverta de le Americhe (Johan Padan à la découverte des Amériques) inclut le temps d'une vie entière dans un espace scénique pratiquement vide ${ }^{\mathrm{r}}$.

Comme à l'époque de Mystère Bouffe, en 1969, Fo se déplace seul, habillé de façon neutre, derrière lui une toile de fond richement illustrée, la même qu'il avait utilisée pour Isabelle, trois caravelles et un charlatan. Le seul objet de scène est un album assez volumineux, placé sur un pupitre, que Fo feuillette de temps en temps en reprenant son souffle. En effet, comme le dit Marisa Pizza, il s'agit bien d'un "souffleur muet ${ }^{2}$ ", un véritable scénario visuel réalisé par l'auteur lui-même avec ses propres dessins.

Dario Fo porte sur scène ses dessins sans les montrer directement, il fait en sorte que la curiosité du spectateur monte jusqu’à la pause entre

I. D. Fo, Johan Padan a la descoverta de le Americhe, dans Teatro, sous la direction de Franca Rame, Turin, Einaudi, coll. "I millenni », 2000.

2. M. Pizza, Il gesto la parola l'azione. Poetica, drammaturgia e storia dei monologhi di Dario Fo, Rome, Bulzoni, I996, p. 299. 
les deux actes pour mettre son grand album en consultation libre dans le foyer du théâtre ${ }^{3}$.

Ce coup semble faire partie d'une stratégie de communication ayant comme but un jeu avec le public : si sur les pages de l'album se succèdent des paysages fantastiques, corps nus qui s'enlacent, porcs, chevaux, sirènes, explosions et cataclysmes, sur scène c'est le rythme de la narration en grammelot ${ }^{4}$ qui construit le spectacle, à l'aide d'une gestualité esquissée.

Le décalage entre ce qui est visible sur scène et ce qui est en dehors de la scène, ne se limite pas à créer un simple effet de suspense, mais vise à provoquer une réaction du public; qu'il s'agisse du déclanchement d'une activité imaginative fertile ou d'une simple envie de vérifier sur un support tangible la cohérence des images picturales avec le récit qu'il vient d'entendre, le spectateur est invité à compléter le conte à partir d'un vide visuel.

Selon le critique Christopher Cairns, le monologue Johan Padan a la descoverta de le Americhe est le résultat d'un processus d'affinement du langage théâtral de Fo qui a lieu dans les années quatre-vingt, une période dans laquelle sa "vision picturale ", dérivante de sa formation et de sa pratique de peintre, se manifeste dans toute son ampleur :

Je suis désormais persuadé - écrit Cairns - que celui qui fait du théâtre, ayant une expérience de peintre derrière lui, regardera toujours la scène comme un peintre. La création d'une scénographie, d'un code performatif au théâtre ou du dessin d'un costume est toujours une action picturale, mais dans laquelle le peintre apporte une expérience unique, une vision d'ensemble, du monde, qui est tout à fait particulières'.

L'étude de Cairns sur le Johan Padan, qui prend appui à son tour sur celui d'Antonio Scuderi' ${ }^{6}$, décrit le code performatif de l'acteur qui reproduit l'image dessinée " dans l'esprit du spectateur ${ }^{7}$ ». Le code figuratif des dessins contenus dans le "souffleur muet » se traduit ainsi en code performatif sur scène dans un « langage scénique mûr [...] éloigné de l'expression naturaliste d'un tableau historique d'époque ${ }^{8} »$.

3. Ibid., p. 297.

4. Avec le mot grammelot nous nous référons au langage onomatopéique utilisé par Fo dans ses monologues.

5. C. Cairns, Dario Fo e la "pittura scenica". Arte teatro regie 1977-1997, Naples, Edizioni Scientifiche Italiane, 2000, p. 13 : "Ormai sono convinto che chi fa teatro con l'esperienza del pittore alle spalle guarderà la scena sempre da pittore. La creazione di una scenografia, di un codice performativo nel teatro o del disegno di un costume è sempre un'azione pittorica, ma nella quale il pittore porta un'esperienza speciale, una visione dell'insieme, del mondo, che è del tutto particolare. " La traduction en français est la nôtre.

6. Voir A. Scuderi, Dario Fo and the Popular Performance, New York-Ottawa-Turin, Legas, I998.

7. C. Cairns, Dario Fo e la "pittura scenica", ouvr. cité, p. I85.

8. Ibid., p. I89 "[...] il linguaggio scenico maturo di Fo fabulatore è lontano dall'espressione naturalistica di un quadro storico d'epoca. " 
Marisa Pizza souligne que Dario Fo conçoit ce monologue en racontant les images que lui-même a peintes, dans un processus de création qui commence avec un canevas initial et qui continue sur scène avec des passages improvisés.

À ce propos, Antonio Scuderi identifie dans ces dessins la récurrence de certaines « images-symboles " qui, à son avis, suggèrent l'action scénique, et des icônes/archétypes typiques de la " peinture scénique " de Fo, synthétisant des lazzis?.

Il s'agit d'une utilisation différente du dessin par rapport aux mises en scène des pièces de Molière à la Comédie-Française à Paris en I990-I99I, lorsque Fo avait comblé les lacunes visuelles du texte théâtral (ou celles qu'il considérait comme telles) avec des croquis.

En regardant les enregistrements des spectacles, nous avons l'impression que dans les farces de Molière mises en scène par Fo, les acteurs et les objets de scène faisaient partie d'un seul continuum dynamique dans lequel les images battaient la mesure de la représentation, alors que dans le monologue Johan Padan, c'est la narration qui donne le rythme au spectacle et l'image - comme le montrent Cairns, Scuderi et Pizza - est suggérée et non représentée.

Dario Fo, à travers Johan Padan, peint sur scène, l'auteur joue la peinture et propose au spectateur de jouer avec lui.

Ce qui nous parait crucial est que cette stimulation de la fonction imaginative du spectateur a lieu parallèlement à la récupération de la « conscience autobiographique ${ }^{\mathrm{IO}}$ " du personnage fabulateur : Johan Padan est effectivement le premier des monologues de Fo dans lequel le protagoniste raconte sa vie de sa jeunesse à sa vieillesse. La fable s'étend ainsi sur le temps historique qui nous fait penser à l' " information traditionnelle » dont parlait Umberto Eco dans son essai Apocalyptiques et Intégrés :

[...] si l'information traditionnelle était pour la plupart d'ordre historique [...], l'homme de l'ère "visuelle" reçoit une masse vertigineuse d'informations sur ce qui est en train de se passer dans l'espace, au détriment des informations sur les évènements temporels ${ }^{\mathrm{II}}$.

La narration orale d'une autobiographie fantastique a l'air d'une provocation supplémentaire pour le public à retrouver le temps historique à travers

9. A. Scuderi, Dario Fo and the Popular Performance, ouvr. cité, p. 57-62.

Io. Nous empruntons cette expression au neurobiologiste portugais Antonio Damasio.

II. U. Eco, Apocalittici e integrati, Milan, Bompiani, 1977, p. 347 : " [...] mentre l'informazione tradizionale era per lo più di ordine storico [...], l'uomo dell'era "visuale" viene ad avere una mole vertiginosa di informazioni su quanto sta avvenendo nello spazio, a detrimento delle informazioni sugli eventi temporali. " 
la voie du conte, d'autant plus que cela révèle avec ironie les paradoxes de l'Histoire, dans une époque caractérisée par l'information en temps réel.

L'attitude de Dario Fo semble ainsi répondre à la suggestion que Eco avait formulée trente ans auparavant : "Une civilisation démocratique se sauvera seulement si elle fera du langage de l'image une provocation à la réflexion critique, non une invitation à l'hypnose. ${ }^{12}$ " L'affinement du langage théâtral dont parle Cairns se situe, à notre avis, dans un cadre de réflexion visant à proposer au public un plaisir autre que l'" hypnose ". Comme l'a dit Ferruccio Marotti en I999, à l'occasion de la remise du diplôme laurea honoris causa à Dario Fo à l'université La Sapienza de Rome, Fo porte une attention constante au rôle du théâtre dans la société :

Dario Fo pense que le théâtre aujourd'hui - dans un monde où le cinéma et la télévision constituent désormais l'univers entier du spectacle et nous enchantent pendant des heures et des heures chaque jour - doit avoir une fonction spécifique et différente : un lieu où réfléchir sur nous-mêmes, activer notre intelligence, notre sens critique, un lieu où nous reconnaître membres actifs d'une société qui tend à nous rendre passifs : bref, un lieu où parler de l'homme d'aujourd'hui' ${ }^{13}$.

Ces mots nous confirment que le travail théâtral de Fo a peut-être connu des changements dans les formes et dans les contenus mais que son intention reste la même ; nous avons l'impression que les modalités de communication proposées par l'auteur dans le Johan Padan constituent une réponse aux changements du contexte de réception.

Pendant les années soixante-dix l'activité de Dario Fo et Franca Rame s'était enracinée dans le milieu ouvrier et étudiant et avait opéré comme outil de contre-information visant à dévoiler la violence et les contradictions du pouvoir. Cette époque était caractérisée par un climat de forte tension politique et sociale et marquée par le fléau du terrorisme, ce qui n'est pas le cas pour les années quatre-vingt, qui représentent un tournant et dans la société italienne et dans leur carrière.

Cairns repère le début de ce tournant en 1977 , quand ils font leur retour sur les plateaux de la télévision après les quinze ans d'absence qui avaient suivi le «scandale de Canzonissima » en novembre $1962^{14}$. À cette occasion,

I2. Ibid., p. 346 : "Una civiltà democratica si salverà solo se farà del linguaggio dell'immagine una provocazione alla riflessione critica, non un invito all'ipnosi."

13. F. Marotti, Elogio di Dario Fo, 2006 : "Dario Fo ritiene che il teatro oggi - in un mondo in cui il cinema e la televisione costituiscono oramai l'intero universo dello spettacolo e ci incantano per ore e ore al giorno - debba avere una funzione specifica e diversa: un luogo in cui riflettere su noi stessi, attivare la nostra intelligenza, il nostro senso critico, un luogo in cui riconoscerci come membri attivi di una società che tende a renderci passivi: insomma, un luogo in cui parlare dell'uomo di oggi."

I4. Le couple Fo-Rame rompit le contrat avec la Rai, à cause de la censure d'un sketch satyrique dénonçant les morts sur le lieu de travail dans le secteur du bâtiment. 
Fo signe un contrat avec la Rai pour la diffusion en deuxième partie de soirée d'un cycle de sept spectacles, enregistrés préalablement, dont seulement un inédit, Parliamo di donne [Récits de femmes], conçu pour Franca Rame. La rétrospective comprenait Isabella, tre caravelle e un cacciaballe [Isabelle, trois caravelles et un charlatan, 1963], Settimo : ruba un po' meno [Septième commandement: tu voleras un peu moins, 1964], deux versions de Ci ragiono e canto [J'y raisonne et je chante, 1966], La Signora è da buttare [Il faut la balancer, cette dame, 1967] et deux versions de Mistero buffo [Mystère bouffe, i969].

Selon Cairns ces spectacles constituaient "un résumé ou un compte rendu du langage théâtral de Fo jusqu'au moment du grand saut dans l'univers de la régie avec Histoire du soldat de Stravinskji [1978-1979] ${ }^{15}$ et avaient tous en commun la "vision picturale " de Fo. Il souligne également que le retour de Fo à la télévision avait inauguré une époque dans laquelle une telle "vision picturale " s'était manifestée dans toute son ampleur, avec les mises en scène des pièces des grands compositeurs et dramaturges européens (L'Opera dello Sghignazzo, libre remaniement de l'Opéra de quat'sous de Brecht, en 1982, Le Barbier de Séville de Rossini en 1987, Le médecin volant et Le médecin malgré lui de Molière en 199I), mais aussi avec Arlecchino en 1985 et Johan Padan a la descoverta de le Americhe.

Pendant les années quatre-vingt Fo semble donc laisser de côté une dramaturgie fondée sur l'urgence de la représentation pour se consacrer, de manière approfondie, aux techniques de la mise en scène et du jeu de l'acteur.

Selon Marina Cappa et Roberto Nepoti, le cycle de spectacles pour la télévision en 1977 peut être considéré un "bilan ", le témoignage " de la diminution progressive des impulsions novatrices de la décennie précédente » et de l'impossibilité de mettre en scène " une succession d'événements dramatiques qui se prêteraient mal à la déformation satirique ${ }^{16}$ ".

La pièce inachevée Il Caso Moro, écrite en 1979 et jamais représentée, est emblématique de ce qui peut être appelé la crise d'un langage de parole ${ }^{17}$. Dans ce texte, le personnage Fo-Aldo Moro n'arrivait pas à achever son

15. C. Cairns, Dario Fo e la "pittura scenica", ouvr. cité, p. 36 " [...] riassunto o resoconto del linguaggio teatrale di Dario Fo fino al punto del gran lancio nel mondo della regia con Storia del soldato di Stravinskji [I978-79]. "

16. Ibid. : "graduale venir meno degli impulsi innovativi del decennio precedente "; " un susseguirsi di eventi drammatici che mal si presta[va]no alla deformazione satirica". Ces phrases de M. Cappa et R. Nepoti ont été citées par C. Cairns.

17. Voir F. Tummillo, Il buffone incatenato. Il caso Moro di Dario Fo, Actes du colloque internationale Littérature et temps des révoltes (Italie, 1967-1980), université Stendhal-Grenoble 3 - ENS Lyon, 2008 (publication en ligne sur : <http://colloque-temps-revoltes.ens-lyon.fr/spip.php?articleI46\#acte>). 
discours et il devenait victime de ses propres mots, comme le reconnaissait l'auteur lui-même dans une interview :

C'est fini le temps où, pour faire Mort accidentelle d'un anarchiste, il suffisait de renverser une situation apparemment obscure, et tout était clair. Maintenant c'est le pouvoir qui fait entièrement la loi sur ce qui est clair et ce qui ne l'est pas. Et toi, si tu veux faire du théâtre, tu dois aussi faire les comptes avec leur science de la représentation politique, beaucoup plus rapide et paradoxale que le théâtre. Avec Moro, on m’a donné une leçon de spectacle ${ }^{18}$.

La «science de la représentation politique » et la représentation théâtrale sont présentées par Fo comme deux systèmes d'images qui entrent en compétition. La représentation politique prévaut car elle a appris à être obscure, rapide et parce qu'elle s'est approprié le paradoxe, un outil cher au théâtre de Dario Fo.

Ce n'est peut-être pas un hasard si Settimo : ruba un po' meno fait également partie de la rétrospective à la télévision, car il est construit sur une situation qui est initialement présentée comme absurde, mais qui s'avère comme réelle dans la charge grotesque du dénouement final.

Dans cette pièce, Fo montre que souvent la réalité dépasse l'imagination et que, dans un contexte de déferlante spéculation sur les terrains, les administrateurs locaux peuvent bien décider de déplacer un cimetière, d'autant plus qu'ils ont acheté des cadavres à l'étranger pour l'élargir.

Ce spectacle semble avoir été inclus dans la rétrospective pour sa structure dramaturgique qui serait plus adaptée aux paradoxes spectaculaires de la politique italienne que le simple renversement farcesque n'arrivait plus à les raconter ${ }^{19}$.

Les médias avaient joué un rôle fondamental dans ce processus de spectacularisation de la politique et il nous paraît fort probable que Dario Fo et Franca Rame se soient interrogés sur la possibilité d'intégrer activement leur théâtre à ce nouveau panorama socioculturel, sans perdre leur identité.

Cairns, en rejoignant la position de Chiara Valentini, souligne que "l'invitation de la part de la Rai à Fo ${ }^{20}$ " était très probablement due à l'affirmation de la gauche italienne aux élections en 1975 et 1976 et à

18. E intanto Fo chiude Moro nel cassetto, dans L'Europeo, 8 novembre 1979: "Sono finiti i tempi in cui per fare Morte accidentale di un anarchico bastava capovolgere una situazione apparentemente oscura, e tutto era chiaro. Ora è interamente il potere a dettar legge su ciò che è chiaro e ciò che non lo è. E tu, se vuoi far del teatro, devi anche fare i conti con la loro scienza della rappresentazione politica, molto più veloce e paradossale del teatro. Con Moro, mi hanno dato una lezione di spettacolo."

19. Ce spectacle se prêtera parfaitement à une actualisation (Settimo: ruba un pó meno $n^{\circ} 2$ ) quinze ans plus tard, en 1992, lors du scandale de Tangentopoli en Italie.

2o. Voir C. Valentini, La storia di Dario Fo, Milan, Feltrinelli, I977. 
l'identification de Rai 2 comme une chaîne de télévision " presque laïque et socialiste ». Il met également en évidence que le fait de confier à la télévision seulement un certain type de spectacles était un signal clair de ce qu'on ne voulait pas rétablir dans le futur :

Mort accidentelle d'un anarchiste, on n'en parle même pas! Et également Faut pas payer! Le premier parce qu'il met en question le sérieux des forces de l'ordre, le deuxième parce qu'il sembla encourager, à l'époque, la désobéissance civile. Pareil pour Guerre du peuple au Chili, parce que trop lié aux faits divers et à la chronique d'une époque et parce qu'en plus il traitait de problèmes à l'étranger. Mort et résurrection d'un pantin, il n'en est pas question, pour des raisons similaires ${ }^{21}$.

À tous ces arguments, qui relèvent à la fois de la valeur artistique que du contenu des spectacles, nous voudrions ajouter un élément d'ordre pratique et technique lié à la transposition du théâtre à la télévision. Nous ignorons les accords (et les éventuels compromis) entre la Rai et le couple Fo-Rame en 1977, mais nous nous demandons si l'un des critères de sélection des spectacles n'a pas été l'efficacité de leur représentation à la télévision. Nous trouvons une présentation des problématiques principales liées à une telle transposition dans le Dictionnaire du théatre de Patrick Pavis :

Alors qu'au théâtre, le spectateur fait lui-même son tri dans les signes de la représentation, à la télévision (comme au cinéma), un fléchage du sens a déjà été effectué pour lui dans le cadrage, le montage, les mouvements de caméra. Pour une émission à partir d'une mise en scène théâtrale, cela implique que la mise en scène filmique a le "dernier mot" pour donner sens au spectacle ${ }^{22}$.

Il n'est pas impossible que l'attention portée à la mimique d'un seul acteur dans Mistero buffo, les beaux tableaux vivants rythmés par la musique populaire dans Ci ragiono e canto ou la scénographie à fort impact visuel dans Isabella, tre caravelle e un cacciaballe, se prête mieux à une version filmée, d'autant plus que l'année I977 était importante pour la télévision italienne, qui passait officiellement du noir et blanc à la couleur, avec toute une série de potentialités communicatives encore à tester.

Il ne s'agit que d'une hypothèse, mais il est intéressant de constater que, au-delà des critères de choix des spectacles, cette expérience de mise en scène à la télévision (partagée peut-être avec des professionnels de la Rai) a eu lieu

2I. C. Cairns, Dario Fo e la "pittura scenica", ouvr. cité, p. 37 : "Morte accidentale di un anarchico neanche parlarne! Neanche Non si paga! Non si paga! Il primo perché pone in questione la serietà della forze dell'ordine, il secondo perché sembrò allora incoraggiare la disubbidienza civile. Neanche La Guerra del popolo in Cile, perché troppo legata a fatti e cronaca di un'epoca, e per di più trattava problemi all'estero. Morte e resurrezione di un pupazzo, neanche per sogno, per motivi simili."

22. P. Pavis, Dictionnaire du théâtre, Paris, Armand Colin, 2003, p. 348. 
au même moment que le "grand saut dans l'univers de la régie » au théâtre. On peut supposer que l'expérience de la télévision a permis à Fo de se regarder autrement, de se familiariser avec un moyen de communication qui a toujours le «dernier mot » et qu' elle lui a appris à dialoguer avec un public invisible, caché derrière un petit écran dans l'intimité du foyer familial.

Tous ces changements ne sont pas négligeables pour un homme de théâtre si attentif aux spectateurs, d'autant plus que ce public invisible était probablement plus ample et hétérogène qu'à l'époque de Canzonissima, et qu'il aurait perdu progressivement, au cours des années quatre-vingt, sa "physionomie de classe " tandis qu'augmentait son sens d'appartenance à la "deuxième révolution de la consommation ${ }^{23}$ ". Cette dernière était encouragée par le grand succès des chaînes de télévision commerciale en Italie, une donnée importante si l'on considère que, comme l'a écrit Andrea Di Michele, la télévision devenait le principal moyen de « consommation culturelle» :

Pour beaucoup d'italiens, et surtout pour les classes inférieures, la télévision devint le principal moyen de consommation culturel, conquérant ainsi le premier rang parmi les loisirs. En 1986, 86,3\% des interviewés déclaraient regarder quotidiennement la télévision, alors que moins de la moitié lisaient un journal avec la même fréquence ${ }^{24}$.

Di Michele souligne également que la télévision publique adapta progressivement ses contenus à ceux de la télévision commerciale pour contraster sa concurrence, baissant ainsi la qualité de ses programmes. À la lumière de tous ces éléments, nous pouvons identifier deux problématiques principales : l'une concernant les caractéristiques structurelles de la télévision en tant que médium, l'autre portant sur le changement du contexte de réception. L'année I977 se révèle ainsi délicate pour le futur du langage théâtral chez Dario Fo. La célèbre phrase de Marshall McLuhan " the medium is the message " est toujours d'actualité, car elle souligne la prééminence du moyen de communication sur le contenu de la communication. Le critique canadien suggérait, dès les années soixante, que chaque médium détermine une forma mentis propre à ses utilisateurs et qu'il doit être étudié à partir des structures sur la base desquelles il organise la communication ${ }^{25}$. En 1964, Umberto Eco, toujours dans son Apocalyptiques et Intégrés, avait déjà parlé des «nouvelles habitudes de réception » crées par la télévision

23. A. Di Michele, Storia dell'Italia repubblicana, Milan, Garzanti, 2008, p. 277.

24. "Per molti italiani, specialmente i ceti inferiori, la televisione divenne il principale consumo culturale, conquistandosi il primato tra i diversi modi di gestione del tempo libero. Nel 1986, l'86,3\% degli intervistati dichiarava di guardare giornalmente la televisione, mentre meno della metà leggeva un giornale con la stessa frequenza. " (Ibid.)

25. M. McLuhan et Q. Fiore, Understanding Media: The Extensions of Man, New-York, McGraw-Hill, 1964. 
en faisant également référence aux théories de Marshall Mc Luhan. Eco focalisait l'attention sur la réaction des intellectuels face à toutes les transformations du contexte médiatique et classait ces derniers - non sans un brin de provocation - en deux catégories, les apocalyptiques et les intégrés:

[...] si les apocalyptiques survivent justement en confectionnant des théories sur la décadence, les intégrés théorisent, et plus facilement agissent, produisent, émettent leurs messages quotidiennement à tous les niveaux ${ }^{26}$.

Umberto Eco est le premier à faire de l'ironie sur l'utilisation rigide de ces deux catégories et nous voudrions suivre la suggestion tacite de cette ironie en évitant de définir Fo parmi les « intégrés ». Mais il est vrai que Fo a tendance à agir et à produire plus qu’à théoriser. Sa biographie, marquée par plusieurs ruptures et recompositions, nous en dit long sur sa nature d'artisan infatigable prêt à se mettre en discussion et à tout refaire quand le spectacle n'a pas suffisamment de prise sur le public. Nous pouvons synthétiser cette attitude avec l'expression "Voilà le truc: toujours jeter tout en l'air ", qui est le titre d'un paragraphe que l'on trouve dans son Manuale minimo dell'attore ${ }^{27}$. Dans ce texte, Fo montre comme exemple de son modus operandi, le processus de construction de sa comédie Claxon, trombette e pernacchi [Klaxons, trompettes et pétarades] en I98I, fortement remaniée plusieurs fois avant la mise en scène. Ce qui nous paraît intéressant c'est que l'auteur avoue clairement avoir identifié, en accord avec Franca Rame, les "structures dépassées " du texte théâtral qu'il venait d'écrire après l'avoir lu publiquement, c'est-à-dire à un stade déjà très avancé du travail. C'est à partir du retour de l'auditoire qu'il décida alors d'y apporter des changements substantiels.

L'attitude de l'auteur face à son ouvre révèle une rare capacité de se mettre en question et une recherche constante d'un langage théâtral efficace qui prend en compte son interlocuteur.

Cette attitude de Fo, toujours visant à construire et à proposer des solutions, a sûrement le point faible de ne pas s'appuyer sur un "système théorique " conçu pour comprendre le contexte de réception, comme il ressort des mots de Claudio Meldolesi en 1978 :

[...] face au choix entre défendre en tant que "principes » les valeurs de son ancienne position militante ou se mesurer avec les pièges de la télévision, [Fo] a pris la deuxième voie, persuadé de la possibilité d'" ouvrir de nouveaux espaces " en s'insinuant

26. U. Eco, ouvr. cité, p. 4: "Se gli apocalittici sopravvivono proprio confezionando teorie sulla decadenza, gli integrati teorizzano, e più facilmente operano, producono, emettono i loro messaggi quotidianamente ad ogni livello. "

27. D. Fo, Manuale minimo dell'attore, Turin, Einaudi, I997, p. I67-I68 : "Il trucco è: buttare sempre tutto all'aria." 
dans les fissures du pouvoir [...] ; sa position intellectuelle n’a pas changé, mais son système théorique, dans le reflux du "mouvement " se révèle partiellement inadéquat et impuissant face aux incursions hégémoniques ou destructives (il y en a déjà et d'autres se préparent) ${ }^{28}$.

La question qui se pose aujourd'hui est de savoir si Fo a effectivement ouvert de nouveaux espaces. Le monologue, transformé en biographie fantastique, constitue, à notre avis, un de ces espaces, un lieu qui peut se dilater ou se contracter, le temps d'un souffle. C'est dans cet espace en mouvement, jamais constant, qui trouve sa place l'image conçue, au sens philosophique, comme « reproduction fantastique, ayant lieu dans la conscience, du contenu d'une expérience sensible ${ }^{29}$ ». D'ailleurs, en regardant les dessins du grand album que Fo porte sur scène avec Johan Padan, on pourra constater que la " reproduction fantastique " est l'approche de Fo aux images picturales des grands peintres classiques qu'il reprend, réinvente, décompose et recompose.

Cette attitude qu'a l'auteur à l'égard de l'Art, est la même qu'il a à l'égard de la Littérature, de l'Histoire et de tout autre savoir écrit avec une majuscule initiale. Dario Fo, avec le Johan Padan, semble vouloir expérimenter un type de provocation autre que celui de la contre-information. Sa "vision picturale », héritage de son passé, retrouve sa force dans un langage théâtral qui veut secouer les spectateurs non seulement sur le plan des contenus, mais aussi sur celui de la modalité de réception de ces contenus. La complicité que Fo veux instaurer avec son public se prolonge hors de la scène à travers la multiplicité de moyens de transmission auxquels il confie désormais son œuvre. Le Johan Padan est né comme un spectacle théâtral et a continué à vivre sur les pages d'une édition illustrée pour ensuite se transformer en dessin animé.

Pour l'instant nous ne sommes pas en mesure de déterminer ni son influence effective sur la manière de faire du théâtre aujourd'hui en Italie ni son impact sur les spectateurs. La force du théâtre de Fo demeure insaisissable pour ceux qui ne partent pas à la découverte des Amériques ${ }^{30}$.

28. C. Meldolesi, Su un comico in rivolta, Rome, Bulzoni, 1978, p. I91 : " [...] posto nell'alternativa di difendere i valori della vecchia posizione militante come "principi" o di misurarsi con l'insidia televisiva, [Fo] ha scelto la seconda via, convinto della possibilità di "aprire nuovi spazi" lavorando nelle crepe del potere [...]; la sua posizione intellettuale non è mutata, mentre il suo sistema teorico, nel riflusso del movimento, appare in parte inadeguato e sguarnito verso le incursioni egemoniche o di devastazione (ce ne sono e se ne preparano)."

29. La piccola Treccani: dizionario enciclopedico, Rome, Istituto della enciclopedia italiana, 1995-1997, définition du mot «Immagine " [Image] : "Con sign. specifico, in filosofia, la riproduzione fantastica, che si compie nella coscienza, del contenuto di un'esperienza sensibile, o la libera produzione di ciò che potrebbe essere il contenuto di una simile esperienza."

30. Je remercie Lisa El Ghaoui et Emanuela Nanni pour leur relecture de cet article et Marisa Pizza pour ses conseils. 